\title{
Preexisting Executive Function Deficits and Change in Health Behaviors During the COVID-19 Pandemic
}

\author{
Bradley M. Appelhans ${ }^{1} \cdot$ Alexis S. Thomas ${ }^{2} \cdot$ Glenn I. Roisman $^{3} \cdot$ Cathryn Booth-LaForce $^{2} \cdot$ Maria E. Bleil $^{2}$
}

Accepted: 22 February 2021 / Published online: 2 March 2021

(c) International Society of Behavioral Medicine 2021

\begin{abstract}
Background Executive functions (EF) support engagement in goal-directed behaviors, including several health behaviors. Stressful and cognitively demanding events can disrupt EFs and interfere with health behavior, possibly to a greater extent in those with preexisting EF deficits. This study examined the association between preexisting EF deficits and subsequent negative changes in eating patterns, physical activity, sedentariness, and alcohol/substance use during the COVID-19 pandemic. Method Participants were 374 young adults in a follow-up study of the longitudinal, multisite Study of Early Child Care and Youth Development (SECCYD). Preexisting EF deficits were assessed with the Barkley Deficits in Executive Function Scales-Short Form, and personally impactful negative changes in four health behaviors (physical activity, unhealthy eating, sedentary time, alcohol/substance use) during the COVID-19 pandemic were subsequently assessed with the EpidemicPandemic Impacts Inventory.

Results In ordered logistic regression models, higher preexisting total EF deficits were associated with greater negative impactful changes in physical activity and unhealthy eating, independent of sociodemographic variables, obesity, and (as relevant) accelerometer-based physical activity and pre-COVID-19 diet quality. Socioeconomic status moderated the association between total EF deficits and impactful change in alcohol/substance use, with EF deficits linked to greater impactful change in alcohol/substance use only in higher socioeconomic strata.

Conclusion Individuals with preexisting EF deficits appear more vulnerable to the negative impact of the COVID-19 pandemic on several key health behaviors. As the pandemic unfolds, strategies may be needed to identify those with EF deficits (e.g., screening tools) and provide them with tailored support for chronic disease risk reduction.
\end{abstract}

Bradley M. Appelhans

brad_appelhans@rush.edu

Alexis S. Thomas

alexist1@uw.edu

Glenn I. Roisman

roism001@umn.edu

Cathryn Booth-LaForce

ibcb@uw.edu

Maria E. Bleil

mbleil@uw.edu

1 Department of Preventive Medicine, Rush University Medical Center, 1700 W. Van Buren St., Suite 470, Chicago, IL 60612, USA

2 Department of Child, Family, and Population Health Nursing, University of Washington, Box 357262, Seattle, WA 98195, USA

3 Institute of Child Development, University of Minnesota, Minneapolis, MN, USA

\section{Introduction}

Unhealthy eating, physical inactivity, and heavy alcohol/substance use confer substantial risk for morbidity and mortality from chronic disease [1-3]. Within dual-process models of health behavior, executive function (EF) is central for supporting adherence to a healthy lifestyle [4-6]. EFs are a set of cognitive processes that support goal-directed behavior, including inhibitory control, working memory, cognitive flexibility, and other second-order processes (e.g., planning, problem-solving) [7]. Individuals with EF deficits have difficulty adhering to a healthy diet, maintaining an active lifestyle, and moderating their alcohol and substance use in modern environments characterized by temptations and competing demands $[8,9]$.

EF can be disrupted by experiences in daily life, leading to a temporary cognitive shift towards habitual/automatic behaviors [10]. For example, stress and increased cognitive demands have been found to produce temporary decrements 
in executive functioning $[11,12]$ and can precipitate lapses in health behaviors $[13,14]$. There is some evidence that individuals with lower baseline EF capacities are more vulnerable to temporary disruptions in EF following stress or cognitive demands [15].

The COVID-19 global pandemic has had a prolonged and significant impact on society, and represents a source of significant stress and cognitive demands for many individuals [16]. It is unknown how an event of this scale can impact health behavior for individuals with and without EF deficits. This study leveraged a well-characterized cohort of young adults to test whether preexisting EF deficits are prospectively associated with impactful changes in key health behaviors during the COVID-19 pandemic. Changes in unhealthy eating, physical activity, sedentariness, and alcohol/substance use since the beginning of the pandemic, and the negative personal impact of these changes, were assessed between June and August 2020, a period in which public health measures such as stay-at-home orders, business and school closures, and travel restrictions were widely implemented. To our knowledge, this is the first study to test whether EF deficits predict the impact of a global pandemic on health behavior change.

\section{Methods}

\section{Participants}

The sample was drawn from the NICHD Study of Early Child Care and Youth Development (SECCYD), a prospective birth cohort of children and their families $(N=1364)$ recruited in 1991 from 10 US cities: Charlottesville, VA; Irvine, CA; Lawrence, KS; Little Rock, AR; Madison, WI; Morganton, NC; Philadelphia, PA; Pittsburgh, PA; Seattle, WA; and Wellesley, MA. The SECCYD sampling strategy consisted of screening mother-infant dyads of babies born at participating hospitals within preselected $24-\mathrm{h}$ intervals. Exclusion criteria included the following: mother was $<18$ years old, non-English speaking, or had a substance use disorder; serious medical problems (mother or infant); family lived $>1 \mathrm{~h}$ away from the study site; child was being given up for adoption; participation in another study; and refusal to be screened. Oversampling on certain characteristics (e.g., single-parent households) was applied to better approximate the population in each 1990 US Census region. SECCYD assessments captured trajectories of health and development throughout childhood and adolescence. An ongoing SECCYD follow-up study-the Study of Health in Early and Adult Life (SHINE)-is conducting additional follow-up assessments with the SECCYD infants, who are now roughly 29 years of age. SHINE includes an in-person health assessment and collection of social and behavioral data. The most recent follow-up assessment occurred between January 2018 and March 2020. All of the 430 SHINE participants who completed this follow assessment were subsequently asked to complete a questionnaire assessing the impact of the COVID19 pandemic between June and August 2020. Of these, 374 $(87.0 \%)$ completed the questionnaire and were included in the present analyses.

\section{Measures}

Impactful Change in Health Behaviors Participants completed the Epidemic-Pandemic Impacts Inventory (EPII) recently developed by Grasso et al. [17]. Participants indicated whether the ongoing COVID-19 pandemic caused increased engagement in four problematic health behaviors: less physical activity or exercise; overeating or eating more unhealthy foods (e.g., junk food); more time sitting down or being sedentary; and increase in use of alcohol or substances. If endorsed, participants rated the negative impact this change, at its worst, had on their life using a 5-point scale from 0 "No negative impact" to 5 "Extreme negative impact." The responses to these two questions were consolidated into a single, three-level variable reflecting impactful change in health behaviors (no impact, mild/moderate impact, high/extreme impact). Given that few participants endorsed high or extreme negative impact of change in alcohol/substance use, this variable was collapsed to two levels (no impact, some impact).

Barkley Deficits in Executive Function Scales-Short Form (BDEFS-SF) The BDEFS-SF [18] is a validated 20-item rating scale that captures deficits in five domains of executive functioning in adults: Emotion regulation, Self-motivation, Self-restraint, Self-organization, and Time management. The five scale scores each have a possible range from 4 to 16 and are summed to yield a Total executive function deficits summary score (possible range 20-80).

Covariates Gender and race (non-Hispanic white, Hispanic or non-white) were assessed via self-report in the SHINE study. Participants self-reported their educational attainment on a nine-category scale ranging from "no high school diploma" to "doctoral degree" and reported their income in 19 categories ranging from $<\$ 5000$ to $\$ 300,000$ or more. Both socioeconomic status (SES) variables were standardized against the rest of the sample, summed, and re-standardized to provide a single composite score of SES. Pre-COVID-19 average daily minutes of moderate/vigorous 
intensity physical activity was derived from a 7-day accelerometry protocol. The presence of obesity was defined based on a body mass index $\geq 30 \mathrm{~kg} / \mathrm{m}^{2}$ derived from measured height and weight. Pre-COVID-19 diet quality was quantified by applying the Healthy Eating Index-2015 (HEI-2015) scoring system to nutrient data derived from three 24-h diet recalls collected through the National Cancer Institute's Automated Self-Administered 24-hour (ASA24) Dietary Assessment Tool. The HEI-2015 quantifies adherence to 13 dietary components within the 2015 Dietary Guidelines for Americans. Component scores are summed to yield a single score, ranging from 0 to 100 , representing overall diet quality.

\section{Analysis}

Ordered logistic regression was used to model the association of executive function deficits and the three-level variables reflecting impactful change in eating, physical activity, and sedentary time. Ordered logistic regression is an extension of standard logistic regression, which models the likelihood of transitioning from the lower to the higher value of a binary outcome. Ordered logistic regression models the likelihood of transitioning from any lower value to the next higher value of an outcome with three of more levels. For three-level outcomes, the resulting odds ratio applies both to the transitions between level 1 and 2, and between level 2 and 3, and can be interpreted as the likelihood of observing a higher level of the outcome for every 1-unit increase in a predictor. The one model predicting impactful change in sedentary time violated the proportional odds assumption. Repeating this analysis with generalized ordered logit models produced identical findings, so the more interpretable results from ordered logistic regression are reported. As the variable for impactful change in alcohol/ substance use had only two level, binary logistic regression was used to model its association with executive function deficits. All models controlled for sociodemographic variables (gender, race, SES), and the presence of obesity (body mass index $\geq 30 \mathrm{~kg} / \mathrm{m}^{2}$ ). Additionally, models predicting impactful change in physical activity and sedentary time adjusted for pre-COVID-19 engagement in MVPA (average min/day) at the prior assessment, and models examining impactful change in eating behavior adjusted for pre-COVID-19 diet quality (HEI-2015 score) at the prior assessment. The first set of models tested associations between Total executive function deficits and impactful change in health behaviors. Associations involving the BDEFS-SF subscales were subsequently examined only for the health behaviors that were associated with Total executive function deficits. A third set of analyses explored interactions of Total executive function deficits with SES, obesity, and the outcome-specific pre-COVID-19 variables. Analyses were performed in Stata 13 (College Station, TX).

\section{Results}

Descriptive Findings Sample characteristics are summarized in Table 1. SHINE participants are predominantly nonHispanic white (79.7\%), and by design, were all roughly 29 years of age at the COVID-19 pandemic assessment (range 28.6 to 29.5 years). Mean BDEFS-SF subscale (Emotion regulation, $\alpha=.90$; Self-motivation, $\alpha=.73$; Self-restraint, $\alpha=.78$; Self-organization, $\alpha=.81$; Time management, $\alpha=.85)$ and Total EF deficits $(\alpha=.91)$ summary scores (Table 1) were very similar to those for 18-34-year olds in the BDEFS normative sample [18]. The proportions of participants reporting impactful change in health behaviors were $27.5 \%$ for increased alcohol/substance use, $51.9 \%$ for unhealthy eating, $55.3 \%$ for less physical activity, and $70.1 \%$ for more sedentary time. Most participants (87.2\%) reported impactful change in at least one health behavior.

Associations with Total Executive Function Deficits Table 2 presents associations between Total executive function deficits summary scores and impactful change in health behaviors. Higher Total executive function deficits summary scores were significantly associated with greater impactful change in both unhealthy eating (OR $=1.04,95 \%$ CI 1.01 , 1.06) and decreased physical activity $(\mathrm{OR}=1.04,95 \% \mathrm{CI}$ $1.02,1.07)$. To contextualize these associations, a 1 standard deviation increase in EF deficits would increase the odds of reporting a higher level of impactful change (from $0=$ no impact to $1=$ mild $/$ moderate impact to $2=\mathrm{high} /$ extreme impact) by $32 \%$ for unhealthy eating, and by $33 \%$ for decreased physical activity. The overall associations of Total executive function deficits scores with impactful change in sedentary time and alcohol/substance use were not significant.

Interactions with SES, Obesity, and Pre-COVID-19 Variables A significant interaction between SES and Total executive function deficits summary score was observed for impactful change in alcohol/substance use $(p=.02)$. Higher Total executive function deficits summary scores were associated with a greater odds of impactful change in alcohol/ substance use for those above the median of SES (OR = $1.07,95 \%$ CI 1.02, 1.12), but not those below the median of 
Table 1 Sociodemographic characteristics and descriptive information on pre-COVID-19 executive function deficits and impactful change in health behaviors during the COVID-19 pandemic $(N=374)$

\begin{tabular}{|c|c|c|}
\hline & $M(\mathrm{SD})$ & $N(\%)$ \\
\hline Female gender & & $213(57.0)$ \\
\hline \multicolumn{3}{|l|}{ Race/ethnicity } \\
\hline Asian & $6(1.6)$ & \\
\hline Black & $33(8.8)$ & \\
\hline Hispanic/Latino & $25(6.7)$ & \\
\hline Non-Hispanic, white & $298(79.7)$ & \\
\hline Non-Hispanic, other & $12(3.2)$ & \\
\hline Education, college degree or higher & & $235(62.8)$ \\
\hline Household income $\geq \$ 100,000 / y$ & & $90(24.1)$ \\
\hline \multicolumn{3}{|l|}{ Impactful change in health behaviors } \\
\hline \multicolumn{3}{|l|}{ Less physical activity } \\
\hline No impact & & $167(44.7)$ \\
\hline Mild/moderate impact & & $129(34.5)$ \\
\hline High/extreme impact & & $78(20.9)$ \\
\hline \multicolumn{3}{|l|}{ More unhealthy eating } \\
\hline No impact & & $180(48.1)$ \\
\hline Mild/moderate impact & & $135(36.1)$ \\
\hline High/extreme impact & & $59(15.8)$ \\
\hline \multicolumn{3}{|l|}{ More sedentary time } \\
\hline No impact & & $112(30.0)$ \\
\hline Mild/moderate impact & & $182(48.7)$ \\
\hline High/extreme impact & & $80(21.4)$ \\
\hline \multicolumn{3}{|l|}{ Increased alcohol or substance use } \\
\hline No impact & & $271(72.5)$ \\
\hline Some impact & & $103(27.5)$ \\
\hline \multicolumn{3}{|l|}{ Pre-COVID-19 BDEFS-SF Executive function deficits } \\
\hline Total executive function deficits summary score & $29.7(8.1)$ & \\
\hline Emotion regulation & $5.8(2.5)$ & \\
\hline Self-motivation & $5.1(1.6)$ & \\
\hline Self-restraint & $5.5(1.9)$ & \\
\hline Self-organization & $5.5(2.0)$ & \\
\hline Time management & $7.9(2.7)$ & \\
\hline Pre-COVID-19 body mass index $\left(\mathrm{kg} / \mathrm{m}^{2}\right)(n=366)$ & $27.4(6.7)$ & \\
\hline Pre-COVID-19 obesity (body mass index $\left.\geq 30 \mathrm{~kg} / \mathrm{m}^{2}\right)(n=366)$ & & $103(28.1)$ \\
\hline Pre-COVID-19 moderate/vigorous physical activity (min/day) $(n=349)$ & $75.2(60.2)$ & \\
\hline Pre-COVID-19 Healthy Eating Index-2015 (0-100 possible) $(n=364)$ & $50.6(10.6)$ & \\
\hline
\end{tabular}

$B D E F S-S F$ Barkley Deficits in Executive Function-Short Form
SES $(\mathrm{OR}=0.99,95 \%$ CI $0.96,1.03)$. No other significant interactions involving SES emerged. Neither the presence of obesity, nor the outcome-specific pre-COVID-19 variables (diet quality, MVPA), moderated associations with Total executive function deficits summary scores in any model.

Associations with Domain-Specific EF Deficits Based on their association with Total executive function deficits, another set of analyses modeled impactful change in unhealthy eating and decreased physical activity from the five domainspecific EF scores (Table 3). Greater deficits in Emotion regulation, Self-organization, and Time management were related to greater impactful change in both of these health behaviors, whereas associations involving self-motivation and self-restraint were not significant.

\section{Discussion}

The vast majority (87.2\%) of young adults in the SHINE cohort reported at least one negative health behavior change during the COVID-19 pandemic, with more than half reporting impactful increases in unhealthy eating, 
Table 2 Associations between pre-COVID-19 total executive function deficit scores and impactful change in health behaviors during the COVID-19 pandemic

\begin{tabular}{|c|c|c|c|}
\hline & OR & $95 \% \mathrm{CI}$ & Sig \\
\hline \multicolumn{4}{|l|}{ Impactful change in physical activity ${ }^{\mathrm{a}}(N=347)$} \\
\hline Pre-COVID-19 SES & 1.04 & $(0.84,1.29)$ & .71 \\
\hline Female gender & 1.38 & $(0.92,2.07)$ & .12 \\
\hline Non-Hispanic white race/ethnicity & 1.26 & $(0.76,2.09)$ & .36 \\
\hline Pre-COVID-19 obesity (BMI $\left.\geq 30 \mathrm{~kg} / \mathrm{m}^{2}\right)$ & 0.69 & $(0.44,1.09)$ & .11 \\
\hline Pre-COVID-19 moderate-vigorous physical activity (min/day) & 1.00 & $(0.99,1.01)$ & .61 \\
\hline Pre-COVID-19 Total executive function deficits summary score & 1.04 & $(1.02,1.07)$ & $<.01$ \\
\hline \multicolumn{4}{|l|}{ Impactful change in unhealthy eating ${ }^{\mathrm{a}}(N=361)$} \\
\hline Pre-COVID-19 SES & 1.00 & $(0.80,1.24)$ & .98 \\
\hline Female gender & 1.68 & $(1.12,2.51)$ & .01 \\
\hline Non-Hispanic white race/ethnicity & 1.48 & $(0.89,2.49)$ & .13 \\
\hline Pre-COVID-19 obesity (BMI $\left.\geq 30 \mathrm{~kg} / \mathrm{m}^{2}\right)$ & 1.47 & $(0.94,2.32)$ & .09 \\
\hline Pre-COVID-19 diet quality (HEI-2015) & 1.01 & $(0.99,1.03)$ & .54 \\
\hline Pre-COVID-19 Total executive function deficits summary score & 1.04 & $(1.01,1.06)$ & $<.01$ \\
\hline \multicolumn{4}{|l|}{ Impactful change in sedentary time ${ }^{\mathrm{a}}(N=347)$} \\
\hline Pre-COVID-19 SES & 1.23 & $(0.98,1.53)$ & .07 \\
\hline Female gender & 1.83 & $(1.22,2.75)$ & $<.01$ \\
\hline Non-Hispanic white race/ethnicity & 0.76 & $(0.45,1.26)$ & .29 \\
\hline Pre-COVID-19 obesity (BMI $\left.\geq 30 \mathrm{~kg} / \mathrm{m}^{2}\right)$ & 0.71 & $(0.45,1.13)$ & .15 \\
\hline Pre-COVID-19 moderate-vigorous physical activity (min/day) & 1.00 & $(0.99,1.01)$ & .15 \\
\hline Pre-COVID-19 Total executive function deficits summary score & 1.02 & $(0.99,1.05)$ & .09 \\
\hline \multicolumn{4}{|l|}{ Impactful change in alcohol/substance use ${ }^{\mathrm{b}}(N=366)$} \\
\hline Pre-COVID-19 SES & 1.32 & $(1.03,1.70)$ & .03 \\
\hline Female gender & 0.68 & $(0.43,1.09)$ & .11 \\
\hline Non-Hispanic white race/ethnicity & 0.92 & $(0.51,1.67)$ & .78 \\
\hline Pre-COVID-19 obesity (BMI $\left.\geq 30 \mathrm{~kg} / \mathrm{m}^{2}\right)$ & 1.11 & $(0.65,1.87)$ & .71 \\
\hline Pre-COVID-19 Total executive function deficits summary score & 1.02 & $(0.99,1.05)$ & .16 \\
\hline
\end{tabular}

aThree-level outcome (no impact, mild/moderate impact, high/extreme impact) modeled with ordered logistic regression

${ }^{\mathrm{b}}$ Two-level outcome (no impact, some impact) modeled with logistic regression physical inactivity, and sedentariness, and roughly one quarter reporting impactful increases in alcohol/substance use. A few recently published reports also describe a substantial negative impact of the pandemic on physical activity, sedentariness, and eating patterns [19-23], with less evidence for a change in alcohol/substance use
[20, 21]. The long-term impact of these changes on health, particularly in terms of chronic disease risk at the population level, warrants follow-up as the pandemic evolves and eventually dissipates.

The hypothesis that preexisting EF deficits would be associated with impactful change in health behaviors during the
Table 3 Associations between domains of pre-COVID-19 executive function deficits and impactful change in physical activity and unhealthy eating during the COVID-19 pandemic

\begin{tabular}{|c|c|c|c|c|c|c|}
\hline & \multicolumn{3}{|c|}{ Physical activity $^{\mathrm{a}}(N=347)$} & \multicolumn{3}{|c|}{ Unhealthy eating $^{\mathrm{b}}(N=361)$} \\
\hline & $\mathrm{OR}$ & $95 \% \mathrm{CI}$ & Sig & OR & $95 \% \mathrm{CI}$ & Sig \\
\hline Pre-COVID-19 Emotion regulation deficits & 1.13 & $(1.04,1.22)$ & $<.01$ & 1.09 & $(1.01,1.18)$ & .03 \\
\hline Pre-COVID-19 Self-motivation deficits & 1.13 & $(1.00,1.27)$ & $<.05$ & 1.09 & $(0.96,1.23)$ & .18 \\
\hline Pre-COVID-19 Self-restraint deficits & 1.10 & $(0.99,1.22)$ & .08 & 1.08 & $(0.98,1.20)$ & .13 \\
\hline Pre-COVID-19 Self-organization deficits & 1.18 & $(1.07,1.31)$ & $<.01$ & 1.15 & $(1.04,1.28)$ & $<.01$ \\
\hline Pre-COVID-19 Time management deficits & 1.09 & $(1.01,1.17)$ & .03 & 1.12 & $(1.04,1.21)$ & $<.01$ \\
\hline
\end{tabular}

${ }^{a}$ Ordered logistic regression controlling for SES, female gender, non-Hispanic white race/ethnicity, preCOVID-19 obesity, and pre-COVID-19 moderate-vigorous physical activity (min/day)

${ }^{\mathrm{b}}$ Ordered logistic regression controlling for SES, female gender, non-Hispanic white race/ethnicity, preCOVID-19 obesity, and pre-COVID-19 diet quality (HEI-2015) 
COVID-19 pandemic was partially supported. Individuals who reported higher preexisting EF deficits endorsed more impactful increases in unhealthy eating and physical inactivity during the pandemic, even while adjusting for their pre-COVID-19 diet quality and physical activity levels, respectively. Preexisting EF deficits were associated with impactful change in alcohol/substance use only for those at higher levels of SES, which may stem from the fact that alcohol and substance use problems are more common in higher socioeconomic strata [24]. The association between preexisting EF deficits and impactful change in sedentariness was smaller in magnitude and did not reach statistical significance, possibly due to the fact that $70 \%$ of the cohort reported impactful change in this outcome, leaving little variance to explain from individual difference variables such as preexisting EF deficits.

Though all five EF deficit domains showed similar magnitude associations with impactful change in physical activity and unhealthy eating, two domains (Self-motivation and Self-restraint) were not statistically significant. It is unclear whether the lack of significance for these associations stems from insufficient power, or indicates that some EF domains are more or less relevant to health behavior change during the pandemic. A fully powered, definitive test of this hypothesis is warranted.

The finding that higher pre-COVID-19 EF deficits confer risk for more impactful negative changes in several important health behaviors during the pandemic is consistent with predictions from dual-process models of health behavior. Adhering to a healthy diet and physical activity regimen, and moderating alcohol/substance use, are thought to be dependent on EFs such as planning and inhibitory control $[4-6,8,9]$. The societal and personal disruptions associated with the COVID-19 pandemic present psychological stressors and cognitive burdens that can disrupt EFs, particularly among those with greater baseline EF deficits. Given the present results, those with EF deficits will likely need additional support to reduce chronic disease risk as the pandemic unfolds. EF deficits can interfere with adherence in the context of behavioral interventions [25-27], and it may be necessary to integrate screening for EF deficits and adapt established interventions to promote health behavior change in this population.

Strengths and Limitations This study leveraged an existing, well-characterized cohort to ascertain the impact of the COVID-19 pandemic on key health behaviors that are known to be dependent on EF. A strength of this study was that EF deficits and baseline markers of health behaviors were measured prior to the COVID-19 pandemic, providing temporality in the observed associations. For some participants, baseline physical activity, obesity, and diet quality were assessed up to 2 years prior to the pandemic and may have changed between the assessments. The restricted age range and ethnic/racial composition of the cohort (formed in 1991) limits the generalizability of results to other populations. EF deficits were assessed via the BDEFS-SR, a well-validated self-report inventory. While the BDEFS is not an objective measure and may be subject to reporting biases, there is evidence that self-report inventories correspond more closely to impairments in daily functioning than do task-based measures, which assess narrow, decontextualized aspects of EF [18, 28-30]. Impactful change in health behaviors was assessed with the recently developed Epidemic-Pandemic Impacts Inventory [17], which could be deployed rapidly for this time-sensitive study, but is not an objective, validated measure. It is almost certain that stay-athome orders issued during the pandemic directly impacted health behaviors for reasons unrelated to EF which were not measured in this study.

Conclusion Individuals with preexisting EF deficits appear to be more vulnerable to the negative impact of the ongoing COVID-19 pandemic on several key health behaviors. The development and dissemination of screening strategies, and interventions tailored to individuals with EF deficits, could help reverse the likely increase in chronic disease risk in this population as the pandemic evolves.

Funding This work was supported by the Eunice Kennedy Shriver National Institute of Child Health and Human Development (U10HD025447, R01HD091132) and the National Heart, Lung, and Blood Institute (R01HL130103) at the National Institutes of Health.

\section{Declarations}

Informed Consent Informed consent was obtained from all individual participants included in the study. All procedures performed in studies involving human participants were in accordance with the ethical standards of the institutional and/or national research committee and with the 1964 Helsinki declaration and its later amendments or comparable ethical standards. This article does not contain any studies with animals performed by any of the authors.

Conflict of Interest The authors declare that they have no conflicts of interest.

\section{References}

1. Morze J, Danielewicz A, Hoffmann G, Schwingshackl L. Diet quality as assessed by the healthy eating index, alternate healthy eating index, dietary approaches to stop hypertension score, and health outcomes: a second update of a systematic review and meta-analysis of cohort studies. J Acad Nutr Diet. 2020;120(1998-2031):e15.

2. Roerecke M, Rehm J. Cause-specific mortality risk in alcohol use disorder treatment patients: a systematic review and metaanalysis. Int J Epidemiol. 2014;43:906-19. 
3. Bott NT, Gargouri F, Chaari L, et al. Dose-response associations between accelerometry measured physical activity and sedentary time and all cause mortality: systematic review and harmonised meta-analysis. Nutrients. 2019;366:14570.

4. Bickel WK, Mellis AM, Snider SE, et al. 21st century neurobehavioral theories of decision making in addiction: review and evaluation. Pharmacol Biochem Behav. 2018;164:4-21 .

5. Dohle S, Diel K, Hofmann W. Executive functions and the selfregulation of eating behavior: a review. Appetite. 2018;124:4-9.

6. Allan JL, Mcminn D, Daly M. A bidirectional relationship between executive function and health behavior: evidence, implications, and future directions. Front Neurosci. 2016;10:386.

7. Diamond A. Executive functions. Annu Rev Psychol. 2013;64:135-68.

8. Hall PA, Fong GT, Epp LJ, Elias LJ. Executive function moderates the intention-behavior link for physical activity and dietary behavior. Psychol Health. 2008;23:309-26.

9. Perry JL, Carroll ME. The role of impulsive behavior in drug abuse. Psychopharmacology. 2008;200:1-26.

10. Schwabe L, Wolf OT. Stress and multiple memory systems: from "thinking" to "doing." Trends Cogn Sci. 2013;17:60-8.

11. Yun RJ, Krystal JH, Mathalon DH. Working memory overload: fronto-limbic interactions and effects on subsequent working memory function. Brain Imaging Behav. 2010;4:96-108.

12. Shields GS, Sazma MA, Yonelinas AP. The effects of acute stress on core executive functions: a meta-analysis and comparison with cortisol. Neurosci Biobehav Rev. 2016;68:651-68.

13. Dunton GF, Rothman AJ, Leventhal AM, Intille SS. How intensive longitudinal data can stimulate advances in health behavior maintenance theories and interventions. Transl Behav Med, in press. https://doi.org/10.1093/tbm/ibz165.

14. Appelhans BM, French SA, Pagoto SL, Sherwood NE. Managing temptation in obesity treatment: a neurobehavioral model of intervention strategies. Appetite. 2016;96:268-79.

15. Otto AR, Raio CM, Chiang A, Phelps EA, Daw ND. Workingmemory capacity protects model-based learning from stress. Proc Natl Acad Sci USA. 2013;110:20941-6.

16. Mcginty EE, Presskreischer R, Anderson KE, Han H, Barry CL. Psychological distress and COVID-19-related stressors reported in a longitudinal cohort of US adults in April and July 2020. JAMA, in press. https://doi.org/10.1001/jama.2020.21231

17. Grasso DJB-G, MJ, Ford JD, Carter AS. The Epidemic - Pandemic Impacts Inventory (EPII). University of Connecticut School of Medicine; 2020.

18. Barkley RA. Barkley Deficits in Executive Functioning Scale (BDEFS). New York, NY: The Guilford Press; 2011.

19. Dunton GF, Wang SD, Do B, Courtney J. Early effects of the COVID-19 pandemic on physical activity locations and behaviors in adults living in the United States. Prev Med Rep. 2020;20:101241.
20. Knell G, Robertson MC, Dooley EE, Burford K, Mendez KS. Health behavior changes during COVID-19 pandemic and subsequent "stay-at-home" orders. Int J Environ Res Public Health. 2020;17:6268.

21. Ammar A, Brach M, Trabelsi K, et al. Effects of COVID-19 home confinement on eating behaviour and physical activity: results of the ECLB-COVID19 International Online Survey. Nutrients. 2020;12:1583.

22. Robinson E, Boyland E, Chisholm A, et al. Obesity, eating behavior and physical activity during COVID-19 lockdown: A study of UK adults. Appetite, in press. https://doi.org/10.1016/j.appet. 2020.104853

23. Meyer J, Mcdowell C, Lansing J, et al. Changes in physical activity and sedentary behavior in response to COVID-19 and their associations with mental health in 3052 US adults. Int J Environ Res Public Health. 2020;17:6469.

24. Vanderlee L, White CM, Kirkpatrick SI, et al. Nonalcoholic and alcoholic beverage intakes by adults across 5 upper-middle- and high-income countries. J Nutr, in press. https://doi.org/10.1093/ jn/nxaa324.

25. Dassen FCM, Houben K, Allom V, Jansen A. Self-regulation and obesity: the role of executive function and delay discounting in the prediction of weight loss. J Behav Med. 2018;41:806-18.

26. Gettens KM, Gorin AA. Executive function in weight loss and weight loss maintenance: a conceptual review and novel neuropsychological model of weight control. J Behav Med. 2017;40:687-701.

27. Mcauley E, Mullen SP, Szabo AN, et al. Self-regulatory processes and exercise adherence in older adults: executive function and self-efficacy effects. Am J Prev Med. 2011;41:284-90.

28. Dehili VM, Prevatt F, Coffman TP. An analysis of the Barkley Deficits in Executive Functioning Scale in a college population: does it predict symptoms of adhd better than a visual-search task? J Atten Disord. 2017;21:567-74.

29. Tarp J, Steene-Johannessen J, Hansen BH, et al. Executive function assessment and adult attention-deficit/hyperactivity disorder: tasks versus ratings on the Barkley Deficits in Executive Functioning Scale. BMJ. 2014;26:1095-105.

30. Barkley RA, Fischer M. Predicting impairment in major life activities and occupational functioning in hyperactive children as adults: self-reported executive function (EF) deficits versus EF tests. Dev Neuropsychol. 2011;36:137-61.

Publisher's Note Springer Nature remains neutral with regard to jurisdictional claims in published maps and institutional affiliations. 